\title{
Microbial Action in Wastewater and Sludge
}

\author{
Agnieszka Mrozik (D)
}

Citation: Mrozik, A. Microbial Action in Wastewater and Sludge. Water 2021, 13, 846. https://doi.org/ $10.3390 / w 13060846$

Received: 4 March 2021

Accepted: 8 March 2021

Published: 19 March 2021

Publisher's Note: MDPI stays neutral with regard to jurisdictional claims in published maps and institutional affiliations.

Copyright: (C) 2021 by the author. Licensee MDPI, Basel, Switzerland. This article is an open access article distributed under the terms and conditions of the Creative Commons Attribution (CC BY) license (https:/ / creativecommons.org/licenses/by/ $4.0 /)$.
Institute of Biology, Biotechnology and Environmental Protection, Faculty of Natural Sciences, University of Silesia, Jagiellońska 28, 40-032 Katowice, Poland; agnieszka.mrozik@us.edu.pl

\section{Introduction}

The global upsurge in urbanization and industrialization is inextricably associated with a systematic increase in the amount of municipal and industrial wastewater, and solid waste. Both sewage and leachate from landfills delivered to sewage treatment plants are often a reservoir of elevated concentrations of contaminants of emerging concern (CECs), such as phenolic compounds, polycyclic aromatic hydrocarbons, pharmaceuticals, dyes, pesticides, cyanides, and heavy metals [1]. Most organic contaminants are characterized by highly diversified structures, low water solubility, poor biodegradability, and environmental persistence. Heavy metal contamination is also an urgent worldwide environmental problem since heavy metals are non-biodegradable and highly toxic [2]. For these reasons, all contaminants may lead to successive deterioration of water quality and adversely impact the ecosystem and human health.

Many wastewater treatment plants face problems with the treatment of wastewater with a high load of dangerous contaminants. Conventional wastewater treatment methods such as activated sludge (AS), sequencing batch reactor (SBR), and membrane bioreactor (MBR) are usually not sufficiently effective in removing CECs. Therefore, it is necessary to establish new solutions that can cope with a complex mixture of compounds and the fluctuations in environmental factors associated with certain types of wastewater.

In this context, the use of different bioremediation processes to remove toxic pollutants from aqueous solutions is gaining considerable attention. Bioaugmentation, the addition of supplementary microorganisms with their associated biodegradation capacities, may allow for an improved performance of the activated sludge process [3]. The candidate bacteria for bioaugmentation should be carefully evaluated with respect to at least three criteria in order to confirm their predispositions to thrive in the conditions for which they were selected and designed to achieve an efficient performance [4]. They should be catabolically able to degrade the individual chemical components in a mixture of contaminants, even in the presence of potentially inhibitory pollutants, persist and compete after their inoculation into a biosystem, and be compatible with autochthonous communities [5]. For the field of bioaugmentation to move forward, it is now essential for key gaps in the research to be addressed. Firstly, the bioaugmentation process must be considered in terms of both the short-term solution to a treatment issue and subsequently the long-term improvement of a system [6]. Unfortunately, current studies tend to focus on laboratory-scale research and synthetic wastewater rather than the practical application of the developed solutions directly in wastewater treatment plants.

Mycoremediation, employing fungi or their derivatives to remove water pollutants, is also considered an effective method for combating the ever-increasing problem of water pollution [7]. Due to their robust growth, vast hyphal network, production of versatile extracellular ligninolytic enzymes, high surface area to volume ratio, resistance to heavy metals, adaptability to fluctuating $\mathrm{pH}$ and temperature, and presence of metal-binding proteins, fungi seem to be ideal candidates for the purification of wastewater and landfill leachate. Fungi use different mechanisms to decontaminate polluted spots and stimulate the environment, including biodegradation, biosorption, and bioconversion of contaminants. Among these methods, the application of fungal biomass as a biosorbent for toxic 
metal remediation has gained particular interest because of its sufficient availability, rapid biosorption/desorption efficiency, and cost competitiveness [8].

\section{Overview of the Special Issue}

This Special Issue consists of five thematically related articles, focusing on various methods to increase the efficiency of pollutant removal from wastewaters and landfill leachates. Among them, two articles present the bioaugmentation potential of selected bacterial strains to improve the aerobic treatment of phenolic landfill leachate $[9,10]$. The third article concerns the bioaugmentation of activated sludge with a bacterial consortium in order to remove selected non-steroidal anti-inflammatory drugs [11], the fourth is a mycoremediation study of old and intermediate landfill leachates [12], and the final review article indicates fungal biosorption as an eco-friendly, economical, and effective method for the removal of harmful pollutants from wastewater [13].

Specifically, Michalska et al. [9] underscored the importance of using a multifaceted approach for selecting suitable candidates to enhance the treatment of wastewater contaminated with phenolic landfill leachate. Moreover, they demonstrated that a genome-centric approach might be useful to complement biochemical tests to select the best strains for specific bioaugmentation. To evaluate the exploitation of ten bacterial strains isolated from various matrices for inoculating AS contaminated with leachate, their degradative potential was analyzed along with their aptitude to synthesize compounds improving the remediation of pollutants in wastewater and their ability to be incorporated into AS flocs. Based on the bacteria's capability to degrade aromatic compounds (primarily catechol, phenol, and cresols) at a concentration of $1 \mathrm{mg} / \mathrm{mL}$ and survive in $12.5 \%$ of the phenolic leachate, Pseudomonas putida OR45a and P. putida KB3 were finally chosen as the best candidates for bioaugmentation of the AS among all of the bacteria tested. Genomic analyses of these two strains revealed the genes encoding enzymes related to the metabolism of aromatic compounds. Additionally, both microorganisms exhibited a high hydrophobic propensity and an ability to produce biosurfactants as well as high resistance to ammonium and heavy metals (especially chromium). Moreover, they exhibited a positive influence on the activated sludge's structure and settleability. These properties enable the exploitation of both bacterial strains in the bioremediation of AS contaminated with phenolic leachate.

In another work by Michalska et al. [10], a bioaugmentation approach was successfully applied in SBRs fed with phenolic landfill leachate by inoculation of AS with two newly isolated Pseudomonas putida OR45a and Pseudomonas putida KB3 strains. According to the results, the SBRs bioaugmented with Pseudomonas strains withstood increasing concentrations of the leachate, which was reflected in the high removal efficiency of the chemical oxygen demand (COD), ammonia nitrogen, and phenolic compounds as compared to the non-inoculated SBR. Simultaneously, the bioaugmentation of the AS allowed the maintenance of high enzymatic activity of dehydrogenases, non-specific esterases, and catalase in this ecosystem, which contributed to a higher functional capacity of indigenous microorganisms than in the non-bioaugmented AS. The stress level experienced by the microorganisms in the SBRs fed with leachate, computed by cellular adenosine triphosphate (ATP) measurements, showed that the abundance of exogenous Pseudomonas strains in the bioreactors contributed to a reduction in effluent toxicity, which was reflected by a decrease in the stress biomass index as compared to the non-bioaugmented AS. This research also emphasizes the successful combination of standard operational parameters with simple and accurate methods (e.g., analyses of the ATP concentration, microbial metabolic activity, and functional potential) instead of focusing on expensive genetic analyses of microbial community structure, as this combination provides early warnings of the performance status of the AS and sewage plant.

Żur et al. [11] also attempted to evaluate the impact of successive bioaugmentation of AS with a defined bacterial consortium consisting of three pharmaceutical-degrading strains, Bacillus thuringiensis B1 (2015b), Stenotrophomonas maltophilia KB2, and Pseudomonas moorei KB4, on the activity and functional capacity of AS microorganisms exposed to 
low concentrations of ibuprofen, naproxen, diclofenac, and paracetamol. Although the addition of the bacterial consortium into the AS did not cause any significant changes in the biomass abundance and metabolic activity of the AS microorganisms, the successive bioaugmentation of the AS caused a slight increase in the metabolic diversity, intensity of carbohydrate usage, and metabolic richness. Microorganisms in the bioaugmented and non-bioaugmented AS were able to degrade the mixture of the analyzed drugs with similar efficiency; however, diclofenac was removed more effectively in the bioaugmented AS. Throughout the experiment, the authors identified a few key secondary metabolites, including hydroxylated derivatives of ibuprofen and diclofenac. The novelty of this work also relates to the isolation and identification of two new bacterial strains Serratia proteamaculans AS4 and Rahnella bruchi AS7, able to degrade almost $0.5 \mathrm{mg} / \mathrm{L}$ of diclofenac, which definitely exceeds the detected environmental concentrations of this drug.

In the next study by Siracusa et al. [12], mycoremediation of old and intermediate landfill leachate with the Ascomycete fungal strain Lambertella sp. was used to remove organic pollutants and toxic compounds. The experiment performed in batch test flasks under co-metabolic conditions, with two different old landfill leachates with suspended and immobilized Lambertella sp. biomass, resulted in a high level of soluble COD depletion. By comparison, intermediate landfill leachate treated in lab-scale reactors operating under continuous conditions for three months, inoculated with immobilized Lambertella sp. biomass, without co-substrates, caused almost total removal of total organic carbon. The myco-based process also reduced the clastogenic effects of the organic fraction of the treated leachates, indicating that Lambertella sp. may be a very promising candidate for the treatment of mature leachates. The authors indicate a non-specific mechanism of depletion for Lambertella sp., which might be related to the production of extracellular laccases.

The great potential of fungal pellets to remove dangerous water pollutants is extensively presented and discussed in the article by Legorreta-Castañeda et al. [13]. In this review, knowledge of the biosorption capacity of fungal pellets towards phenolic compounds, dyes, humic substances, pesticides, pharmaceuticals, and heavy metals, after a short presentation of the pollutants considered, was synthesized. The biosorption performance of fungal pellets is attracting growing attention since they offer process advantages over the culture of dispersing mycelia, such as enhanced biomass separation and high resilience under harsh environmental conditions. Finally, after careful recognition of the adsorption capacity of pellets, their removal efficiency, and the operational conditions, the authors emphasized that the biosorption of complex mixtures of pollutants should be explored more than that of individual chemicals, as well as recommending that more studies should be done with real wastewater and at pilot scale and large scale. It is also important to develop the post-treatment of fungal biomass after treatment of water pollutants.

\section{Conclusions}

This Special Issue provides valuable results and new views on the topic of pollutant removal from wastewater and landfill leachate based on various remediation approaches and literature data, including important key challenges and limitations. The novelties worth emphasizing are: the proposition of ideal candidates for bioaugmentation of activated sludge fed with phenolic leachate; the isolation and identification of new diclofenacdegrading strains; the first use of active biomass and stress biomass indices to assess the impact of leachate and bioaugmentation on AS performance; the use of Ascomycete Lambertella sp. in the depletion of the recalcitrant to biodegradation loads of intermediate and old leachate; as well as an interesting presentation of scientific views on pollutant biosorption using fungal pellets. All authors also emphasized the need for further research and indicated future study directions.

Funding: This editorial received no external funding.

Data Availability Statement: Data sharing not applicable. 
Acknowledgments: The Guest Editor would like to thank all authors for submitting their articles and all reviewers for their work and valuable comments. A special thanks goes to the Assistant Editor, Alexandra Wang, for providing invaluable help, constant contact, and successful collaboration.

Conflicts of Interest: The author declares no conflict of interest.

\section{References}

1. Kim, S.; Chu, K.H.; Al-Hamadani, Y.A.J.; Park, C.M.; Jang, M.; Kim, D.-H.; Yu, M.; Heo, J.; Yoon, Y. Removal of Contaminants of Emerging Concern by Membranes in Water and Wastewater: A Review. Chem. Eng. J. 2018, 335, 896-914. [CrossRef]

2. Tchounwou, P.B.; Yedjou, C.G.; Patlolla, A.K.; Sutton, D.J. Heavy Metal Toxicity and the Environment. Exp. Suppl. 2012, 101, 133-164. [CrossRef] [PubMed]

3. Semrany, S.; Favier, L.; Djelal, H.; Taha, S.; Amrane, A. Bioaugmentation: Possible Solution in the Treatment of Bio-Refractory Organic Compounds (Bio-ROCs). Biochem. Eng. J. 2012, 69, 75-86. [CrossRef]

4. Singer, A.C.; van der Gast, C.J.; Thompson, I.P. Perspectives and Vision for Strain Selection in Bioaugmentation. Trends Biotechnol. 2005, 23, 74-77. [CrossRef] [PubMed]

5. Herrero, M.; Stuckey, D.C. Bioaugmentation and Its Application in Wastewater Treatment: A Review. Chemosphere 2014, 140, 119-128. [CrossRef] [PubMed]

6. $\quad$ Raper, E.; Stephenson, T.; Anderson, D.R.; Fisher, R.; Soares, A. Industrial Wastewater Treatment through Bioaugmentation. Process Saf. Environ. Prot. 2018, 118, 178-187. [CrossRef]

7. Akhtar, N.; Amin-ul Mannan, M. Mycoremediation: Expunging Environmental Pollutants. Biotechnol. Rep. 2020, 26, e00452. [CrossRef] [PubMed]

8. Verma, T.; Maurya, A.; Tripathi, M.; Garg, S.K. Mycoremediation: An Alternative Treatment Strategy for Heavy Metal-Laden Wastewater. In Developments in Fungal Biology and Applied Mycology; Satyanarayana, T., Deshmukh, S., Johri, B., Eds.; Springer: Singapore, 2017. [CrossRef]

9. Michalska, J.; Piński, A.; Żur, J.; Mrozik, A. Selecting Bacteria Candidates for the Bioaugmentation of Activated Sludge to Improve the Aerobic Treatment of Nadfill Leachate. Water 2020, 12, 140. [CrossRef]

10. Michalska, J.; Piński, A.; Żur, J.; Mrozik, A. Analysis of the Bioaugmentation Potential of Pseudomonas putida OR45a and Pseudomonas putida KB3 in the Sequencing Batch Reactors Fed with the Phenolic Landfill Leachate. Water 2020, 12, 906. [CrossRef]

11. Żur, J.; Michalska, J.; Piński, A.; Mrozik, A.; Nowak, A. Effects of Low Concentration of Selected Analgetics and Successive Bioaugmentation of the Activated Sludge on Its Activity and Metabolic Diversity. Water 2020, 12, 1133. [CrossRef]

12. Siracusa, G.; Yuan, Q.; Chicca, I.; Bardi, A.; Spennati, F.; Becarelli, S.; Levin, D.B.; Munz, G.; Petroni, G.; Di Gregorio, S. Mycoremediation of Old and Intermediate Landfill Leachates with an Ascomycete Fungal Isolate, Lambertella sp. Water 2020, 12, 800. [CrossRef]

13. Legorreta-Castañeda, A.J.; Lucho-Constantino, C.A.; Beltrán-Hernández, R.I.; Coronel-Olivares, C.; Vázquez-Rodríguez, G.A. Biosorption of Water Pollutants by Fungal Pellets. Water 2020, 12, 1155. [CrossRef] 